\title{
Conception et fonctionnement des adductions par canalisations sous pression à forts débits
}

\author{
par J. Blanc \\ Ingénieur à la Socièté du Canal de Provence \\ et d'Aménagement de la Région provençale, \\ Le Tholonet
}

\section{Introduction}

Dans un aménagement hydraulique d'une certaine importance, on peut distinguer diverses catégories d'ouvrages, de l'amont vers l'aval, du point de vue transport de l'eau à partir de la source, constituées généralement d'un ouvrage de prise sur un cours d'eau:

- les ouvrages d'amenée ou de transport;

- les ouvrages d'adduction;

- les ouvrages de distribution.

Les ouvrages de transport à gros débit sont des canaux ou des galeries suivant les particularités topographiques de la région.

Les ouvrages de distribution sont des réseaux ramifiés de canalisations sous pression qui aboutissent aux prises des utilisateurs.

Il existe presque toujours, entre ces deux catégories d'ouvrages, une catégorie intermédiaire que nous regrouperons sous le terme d' "Adduction ».

Elles sont constituées de canalisations de gros diamètres, de longueur appréciable, fonctionnant souvent sous des pressions élevées, pour des débits de l'ordre de quelques mètres cubes par seconde.

Leurs caractéristiques sont commandées par la topographie de la zone; leur alimentation sera, soit gravitaire si les ouvrages de transport dominent suffisamment la zone, soit par pompage dans le cas contraire.

La S.C.P. possède un certain nombre d'adductions de ce type (environ $65000 \mathrm{~m}$ ) et d'autres sont prévues: BerreOuest, Berre-Nord, Extensions d'Aix-Nord, Toulon-Est, etc., toutes schématisées sur la figure 1. Dans tous les cas, leur projet soulève un certain nombre de problèmes spécifiques que nous examinerons successivement.

\section{Tracé des canalisations}

L'objectif, à savoir la fourniture de débits donnés en un nombre limité de points, doit être obtenu en recherchant le tracé le plus économique qui les relie à la source d'eau.

La solution théorique du problème est assez bien connue et nous n'y reviendrons pas.

La S.C.P. a écrit un programme de calcul sur ordinateur qui permet d'optimiser un tracé de réseau, dont les points sont définis par coordonnées. Ce programme s'enchaine avec celui du calcul des diamètres. Deux ou trois boucles sont nécessaires pour obtenir le résultat à partir d'un premier tracé donné a priori. Mais celui-ci ne tient pas compte des obstacles rencontrés sur le terrain.

Le projeteur se pose alors les questions suivantes : doit-on traverser les parcelles agricoles, pinèdes, zones rocheuses ou inondées ou les contourner?

$\mathrm{Si}$, pour des canalisations de petits diamètres, ces contraintes sont souvent prépondérantes et imposent un tracé en bordure de parcelles, ce n'est plus le cas pour des canalisations de gros diamètre pour lesquelles le tracé le plus direct est toujours préférable.

Pour fixer les idées nous avons calculé le coût des tracés $A C$ et $A B C$ de la figure ci-après, correspondant à une zone rocheuse et à un verger.

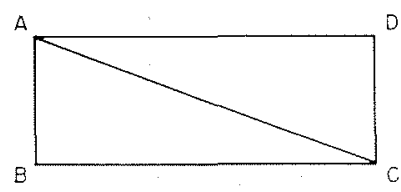




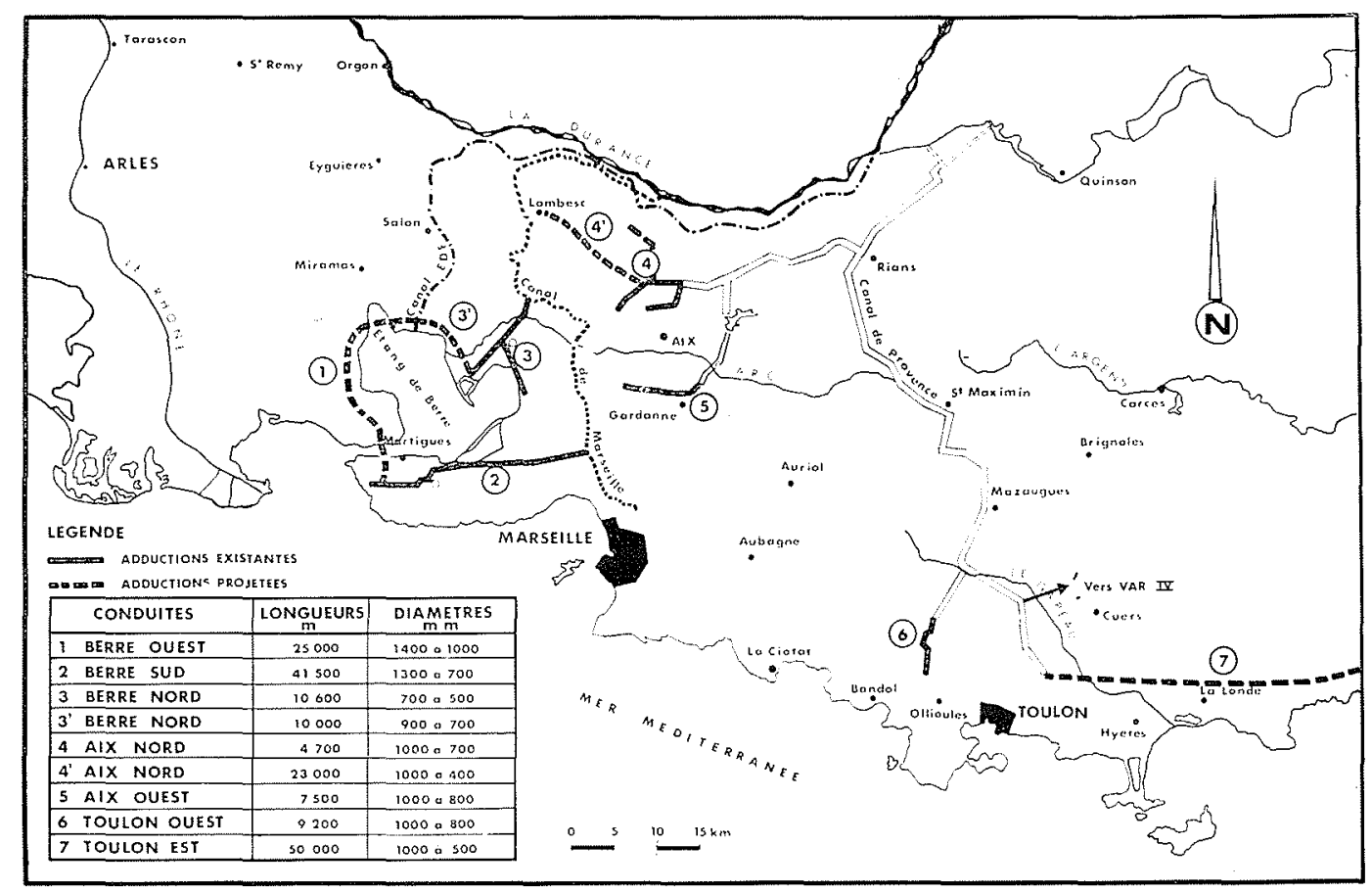

1/ Plan de situation des adductions de la S.C.P.

Le tableau ci-dessous donne la valeur du rapport des longueurs $\mathrm{AB} / \mathrm{BC}$ à partir duquel le tracé diagonal est plus économique.

\begin{tabular}{|c|c|c|}
\hline DiAMĖtre des CANALISATIONS & VIERger & TERrain ROCHEUX \\
\hline 1000 à $1300 \mathrm{~mm}$ & $0,02-0,03$ & 0,14 \\
\hline
\end{tabular}

La faible valeur de ce rapport montre que le tracé diagonal est pratiquement préférable dans tous les cas.

\section{Choix du débit d'équipement en relation avec l'installation éventuelle de réservoirs de compensation}

La plupart des conduites d'adduotion de la S.C.P. assurent un service agricole qui est très variable dans le courant d'une journée de pointe.

La figure 2 illustre ces variations pour le périmètre de Berre-Sud où le débit moyen journalier $Q_{m}$ est égal à $54 \%$ du débit de pointe $Q_{p}$.

$$
Q_{m}=0,54 Q_{p} \text {. }
$$

Deux conceptions du projet sont possibles:

- soit réaliser un ouvrage capable de porter le débit maximum;

- soit écrêter les pointes de débit à l'aide d'une réserve de compensation placée le plus loin possible de la source sur le tracé de la conduite et calibrer cette dernière pour porter le débit moyen journalier.
Dans ce cas, et pour la loi d'évolution des débits donnée ci-dessus, le volume de compensation utile s'exprime en fonction du débit de pointe par:

$$
V=8650 Q_{p} . \quad\left(V, m^{3} ; \quad Q_{p}, \mathrm{~m}^{3} / \mathrm{s}\right)
$$

La deuxième solution est généralement la plus économique. Elle présente en outre l'avantage de prélever sur la source un débit plus régulier.

On pourra se faire une idée de l'utilité d'une réserve de compensation en se reportant aux courbes de la figure 3 donnant les prix 1973, tout compris ouvrage fini, d'une part d'un kilomètre de conduite en fonction de la perte de charge admissible et du débit transporté (voir annexe 1), et, d'autre part, d'une réserve de compensation (déduite des réalisations S.C.P.; voir fig. 4).

Un exemple de calcul est donné ci-après pour une adduction de $16000 \mathrm{~m}$, véhiculant un débit moyen journalier de $0,5,2$ et $4 \mathrm{~m}^{3} / \mathrm{s}$, avec une perte de charge de $2,8 \mathrm{~m} / \mathrm{km}$ (perte de charge égale à celle qui existe sur le feeder de

\begin{tabular}{|c|c|c|c|c|c|c|}
\hline \multicolumn{2}{|c|}{$\begin{array}{c}\text { EQUIPEMENT } \\
Q_{m} \\
\end{array}$} & \multicolumn{2}{|c|}{$\begin{array}{c}\text { EQUIPEMENT } \\
Q_{p} \\
\end{array}$} & \multirow{2}{*}{$\mid \begin{array}{c}P_{m}-P_{p^{3}} \\
\left(10^{3} \mathrm{~F}\right)\end{array}$} & \multirow{2}{*}{$\begin{array}{l}\text { VOLUME } \\
\text { DE } \\
\text { COMPEN- } \\
\text { SATION } \\
\left(\mathrm{m}^{3}\right)\end{array}$} & \multirow{2}{*}{$\begin{array}{c}\text { PRIX } \\
\text { DE LA } \\
\text { RÉSERVE } \\
\left(10^{3} \mathrm{~F}\right)\end{array}$} \\
\hline$\underset{\left(\mathrm{m}^{3} / \mathrm{s}\right)}{Q_{m}}$ & $\begin{array}{c}P_{m} \\
\left(10^{3} \mathrm{~F}\right)\end{array}$ & $\begin{array}{c}Q_{p} \\
\left(\mathrm{~m}^{3} / \mathrm{s}\right)\end{array}$ & $\begin{array}{c}P_{p} \\
\left(10^{3} \mathrm{~F}\right)\end{array}$ & & & \\
\hline 0,5 & 8800 & 0,92 & 12800 & 4000 & 8000 & 880 \\
\hline 2 & 21600 & 3,70 & 32000 & 10400 & 32000 & 1900 \\
\hline 4 & 33600 & 7,40 & 50500 & 16900 & 64000 & 2800 \\
\hline \multicolumn{7}{|c|}{$\begin{array}{l}Q_{m}=\text { Débit moyen; } \\
Q_{p}=\text { Débit de pointe; } \\
P_{m}=\text { Prix correspondant } \\
P_{p}=\text { Prix correspondant }\end{array}$} \\
\hline
\end{tabular}
Berre-Sud). 
Rapport $\frac{\text { Débit }}{\text { Débit de pointe }}$

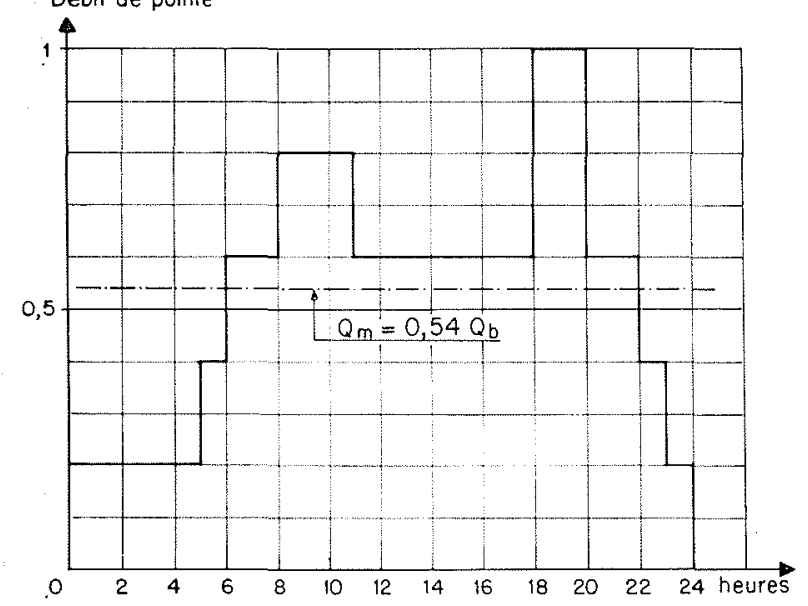

Dans chaque oas, le prix du réservoir est largement compensé par la différence des investissements entre le prix des deux canalisations.

En réalité, une grosse adduction assure souvent un service mixte, à la fois agricole et industriel, et les variations journalières de débit ne concernent qu'une fraction du débit transporté. C'est le cas de l'adduction de Berre-Sud qui assure le transport d'un débit continu de $2,7 \mathrm{~m}^{3} / \mathrm{s}$ et un débit variable $Q_{m}=1 \mathrm{~m}^{3} / \mathrm{s}$.

L'existence du débit continu diminue l'écart entre les prix d'une conduite équipée pour la pointe ou pour le débit moyen, mais ne change pas la conclusion.

Ainsi, pour la conduite de Berre-Sud, l'écart est de $3500000 \mathrm{~F}$, en faveur de la solution avec réserve.

Bien entendu, chaque conduite est un cas d'espèce.

Plus la longueur est importante, plus la solution avec

2)

2/ Courbe $a / Q_{p}=f(t)$ de la zone de Berre Sud.

3/ Prix de la conduite $=f$ (débit, perte de charge).

4/ Prix des réserves au sol.

$P=6500 \mathrm{~V} 0,55$. (Bassins réalisés par édification de digues et revêtement total en dalles de béton).

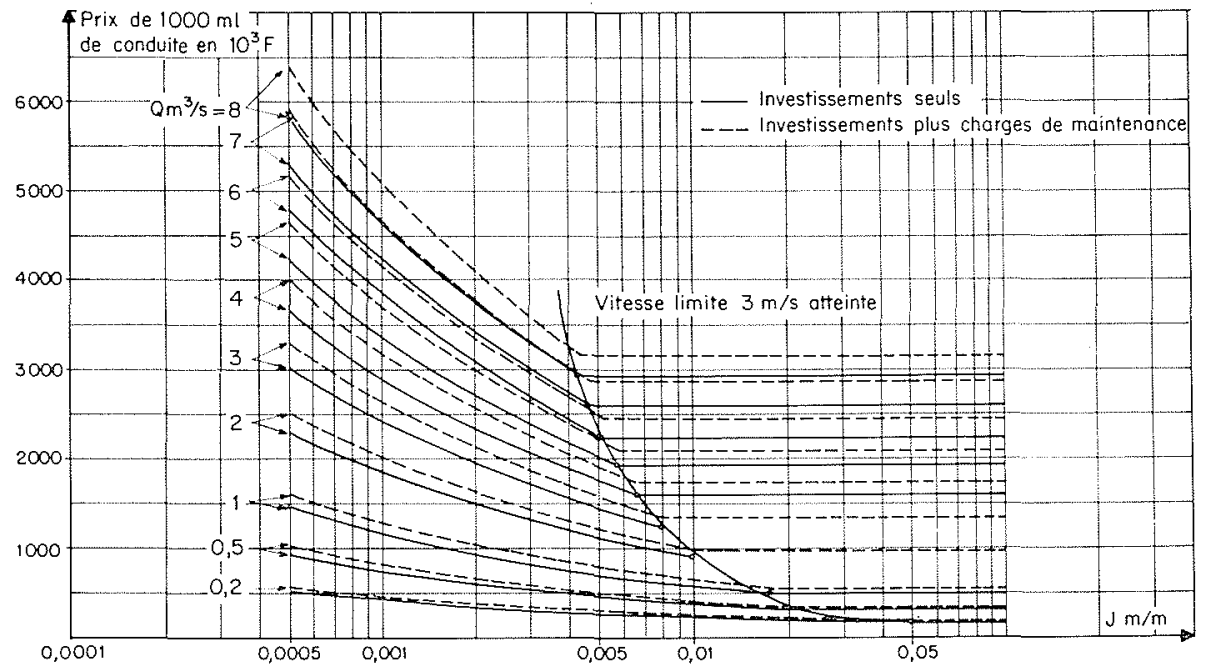

$3 /$

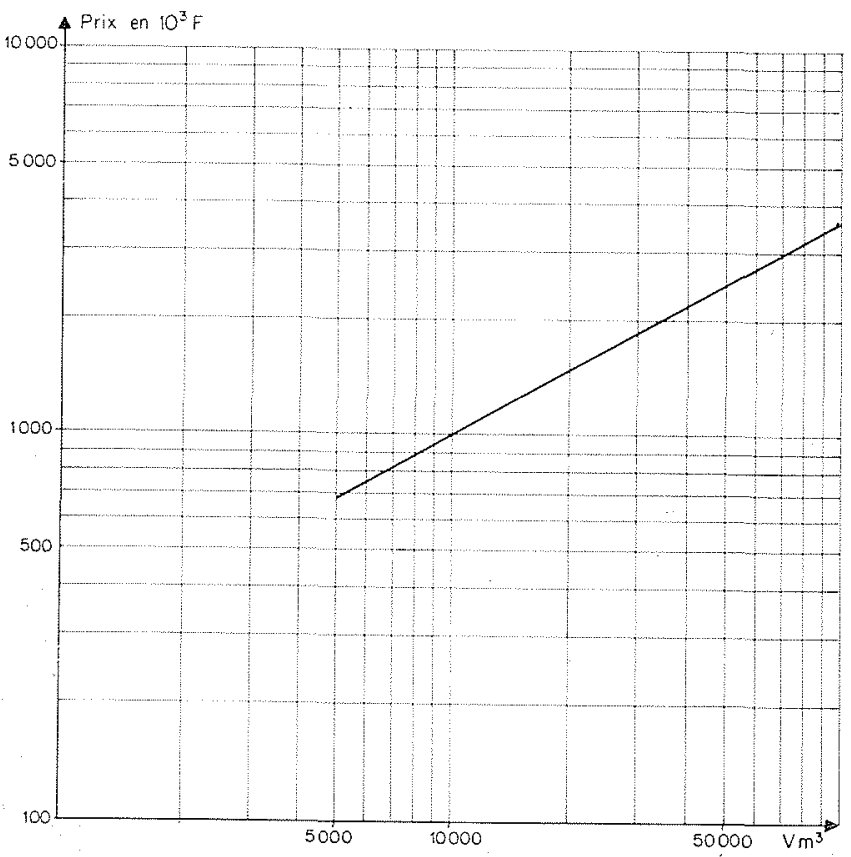

4/ réserve s'impose. Pour fixer les idées, on peut dire que le problème du choix se pose en-dessous de $5000 \mathrm{~m}$ environ.

La perte de charge admissible a également une influence, mais dans les limites habituellement rencontrées, sa valeur n'agit pas considérablement sur le résultat.

\section{Charge disponible Installation éventuelle de surpresseurs}

Le coût des conduites décroît en fonction de la perte de charge disponible. On peut done se demander dans quelle mesure l'installation d'un surpresseur, augmentant cette charge, ne permet pas de réaliser une installation économique.

Pour répondre à cette question, il faut comparer :

- une installation gravitaire, chère en investissements et peu coûteuse en frais d'exploitation et de maintenance; 
- une installation avec surpresseur, plus ou moins chère en investissements et beaucoup plus coûteuse en frais d'exploitation et de maintenance.

Dans la pratique, les calculs se font à l'ordinateur en optimisant :

- d'une part la conduite gravitaire;

- d'autre part l'ensemble surpresseur-conduite.

Les calculs définissent les diamètres des conduites et la hauteur de refoulement économique, et chiffrent chaque solution.

On peut toutefois dégrossir le problème en utilisant les courbes de prix conduites, données ci-dessus, et surpresseurs + frais d'énergie, jointes ci-après (fig. 5 ; cf. annexe 3 ).

\section{Prix des surpresseurs}

Il est très difficile d'évaluer le prix d'un surpresseur par une formule simple. Toutefois, nous pouvons dire que le prix d'un surpresseur (raccordement électrique, taxes et honoraires compris) est proportionnel à sa puissance.

$$
P_{s}=25000 Q H \quad\left(P \text { en } \mathrm{F}, Q \text { en } \mathrm{m}^{3} / \mathrm{s}, H\right. \text { en m) }
$$

Les frais de maintenance sur un tel ouvrage sont élevés. Exprimés en pourcentage de l'investissement, ils sont de l'ordre de $3 \%$ par an, et en valeur actualisée, avec un taux d'actualisation de $10 \%$, ils représentent environ $30 \%$ du coût de l'ouvrage, soit pour un total investissements charges :

$$
P_{s}=32500 Q H \quad\left(P \text { en } \mathrm{F}, Q \text { en } \mathrm{m}^{3} / \mathrm{s}, H \text { en } \mathrm{m}\right)
$$

\section{Dépense d'énergie}

La dépense d'énergie est fonction du rendement global de l'installation, des tarifs de l'énergie et surtout du volume d'eau pompé.

Si l'on admet, en première approximation, que le rendement global est de $70 \%$, que le prix moyen pondéré de l'énergie est de $0,08 \mathrm{~F} / \mathrm{kWh}$, et que l'installation fonctionne en régime continu toute l'année, la dépense d'énergie s'exprime par:

$$
P_{e}=10^{4} Q H \quad(P \text { en } \mathrm{F}, Q \mathrm{en} \mathrm{m} / \mathrm{s}, H \text { en } \mathrm{m})
$$

mais cette dépense a la dimension d'une annuité : elle doit être capitalisée pour être comparée aux investissements.

D'autre part, il est fréquent que la pleine consommation ne soit pas atteinte dès la mise en service de l'installation, ce qui diminue la valeur des dépenses (annuelles et capitalisées) et, pour cette dernière, d'autant plus que le loyer de l'argent est élevé.

Enfin, l'évolution des débits conduit pendant un certain temps à des pertes de charge réduites, donc à des économies sur les frais de pompage; ceci suppose que les pompes peuvent s'adapter à des variations importantes de hauteur de refoulement, ce qui est techniquement, et dans certaines limites, réalisable avec des pompes à vitesse variable.

Lorsque la pleine consommation est atteinte dès la première année de mise en service de l'installation, la dépense d'énergie capitalisée avec un taux de $10 \%$ s'exprime par:

$$
P_{e}=10^{5} Q H \quad\left(P \text { en } \mathrm{F}, Q \text { en } \mathrm{m}^{3} / \mathrm{s}, H \text { en } \mathrm{m}\right)
$$

Lorsque les besoins évoluent dans le temps et que le surpresseur s'adapte aux variations de pertes de charge, cette dépense est inférieure et égale à 30 à $40 \%$ du chiffre ci-dessus, voire inféricure si la conduite dessert des zones agricoles; nous retiendrons la valeur moyenne suivante:

$$
P_{e}=35000 Q H \quad\left(P \text { en } \mathrm{F}, Q \text { en } \mathrm{m}^{3} / \mathrm{s}, H \text { en } \mathrm{m}\right)
$$

Les dépenses concernant le surpresseur et les frais d'énergie, ainsi calculées sont exprimés par la formule:

$$
P_{s}=67500 Q H \quad\left(P \text { en } \mathrm{F}, Q \text { en } \mathrm{m}^{3} / \mathrm{s}, H \text { en } \mathrm{m}\right)
$$

oul encore :

$$
\left.P_{s}=67500 \underset{(P \text { en } F, Q}{Q j} \text { en }^{3} \mathrm{~m}^{3} / \mathrm{s}, j \text { en } \mathrm{m} / \mathrm{m}, L \text { en } \mathrm{m}\right)
$$

Le graphique ci-joint traduit ce résultat sous forme d'abaque pour une longueur de conduite de $1000 \mathrm{~m}$. Associé au graphique concernant les conduites, il permet de répondre rapidement à la question de savoir s'il est opportun de prévoir un surpresseur.

\section{EXEMPLE :}

Une adduction gravitaire transporte un débit de $3 \mathrm{~m}^{3} / \mathrm{s}$ avec une perte de charge de $3 \mathrm{~m} / \mathrm{km}$, peut-on la réaliser plus économiquement avec un surpresseur?

Le prix du kilomètre d'adduction gravitaire est de $1850000 \mathrm{~F}$.

Ce prix devient égal à $1300000 \mathrm{~F}$ si la perte de charge disponible est de $10 \mathrm{~m} / \mathrm{km}$; en augmentant la charge de $7 \mathrm{~m} / \mathrm{km}$, les investissements conduites sont diminués de $550000 \mathrm{~F}$, et la dépense correspondant au surpresseur qui fournit $7 \mathrm{~m} / \mathrm{km}$ est de $1400000 \mathrm{~F}$.

La solution gravitaire est donc plus avantageuse. Le calcul peut être poursuivi pour d'autres points.

Ce problème a été étudié pour l'adduction de Berre-Sud. Le résultat obtenu a également été favorable à l'adduction gravitaire.

Cette conclusion est d'ailleurs assez générale; toutefois, dans certains cas où le passage des points hauts nécessite l'installation d'un surpresseur pour obtenir la hauteur géométrique voulue, l'optimisation de l'ensemble conduitesurpresseur amène à majorer la hauteur géométrique pour atteindre l'optimum de l'ensemble. C'est le cas d'une adduction en projet, Berre-Ouest, où la solution économique est obtenue en déplaçant le surpresseur vers l'amont et en prévoyant celui-ci à vitesse variable.

La dépense d'énergie avec un surpresseur à vitesse variable est environ deux fois plus faible qu'avec un surpresseur à vitesse fixe.

\section{Réalisation en tranches Doublage}

La demande évolue en général lentement et il est souhaitable d'adapter les investissements à cette progression. Le projeteur est ainsi amené à prévoir un fractionnement de l'ouvrage définitif en plusieurs tranches; le plus souvent deux. 
Outre le fait de diminuer les investissements de première phase, cette solution présente l'avantage:

- de pouvoir adapter les ouvrages de doublage à l'évolution réellement constatée et de corriger éventuellement les prévisions initiales;

- d'augmenter la sécurité de l'adduction.

Elle présente, par contre, l'inconvénient d'un encombrement plus important du sous-sol, et l'on sait combien les propriétaires sont sensibilisés par le passage périodique des entreprises sur leur terrain (l'emprise nécessaire pour les travaux de pose d'une conduite de $1000 \mathrm{~mm}$ et plus est de $20 \mathrm{~m})$.

Dans le cas du doublage, le Maître d'ouvrage peut:

- soit adopter le même tracé pour les deux conduites et acquérir une double servitude ( 2 fois $3 \mathrm{~m}$ ) dès la mise en place de la première conduite;

- soit prévoir un tracé différent pour la seconde conduite et reporter à une date ultérieure la prise de servitude.

Cette deuxième solution est moins bonne que la première car, d'une part le tracé le plus économique est celui retenu pour la première conduite, et d'autre part l'occupation du sol avec le temps se réalise toujours dans le sens d'une multiplication des obstacles (routes, habitations, etc.), et il sera toujours de plus en plus difficile de réaliser un tracé direct.

Les échelonnements des investissements et la sécurité font généralement préférer une solution en deux tranches.

Les courbes de la figure 6 montrent dans quelle condition le doublage est valable économiquement.

Pour une conduite de $2 \mathrm{~m}^{3} / \mathrm{s}$ et le taux actuel préconisé par le VI Plan français ( $10 \%$ ), le doublage est économique s'il est réalisé entre 5 et 7 ans après la pose de la première conduite suivant la perte de charge admissible (entre 0,05 et $0,0005 \mathrm{~m} / \mathrm{m}$ ).

On constate, sur ces courbes, que la perte de charge a peu d'influence sur le résultat dès quie le débit est élevé; par contre, le taux dactualisation en a beaucoup.

Ces calculs approchés ont été présentés pour permettre de juger rapidement de l'intérêt d'un doublage et pour dégager les grands principes.

Dans la pratique, le problème n'est pas aussi simple, car les conduites d'adduction desservent plusieurs points de livraison, et ne transportent pas un débit constant sur toute leur longueur.

Les calculs se font alors à l'ordinateur, à l'aide d'un programme de calcul qui étudie et chiffre toutes les solutions possibles en fonction de l'année choisie pour réaliser le doublage, et des courbes d'évolution de la demande de chaque point de livraison, les conduites mises en place en première phase n'étant pas forcément calibrées pour véhiculer un débit moitié du débit exhaustif.

Le projeteur choisit alors, soit la solution conduisant au coût total actualisé minimum, soit la solution nécessitant le plus faible investissement initial et dont le coût total actualisé s'écarte peu (pourcentage à fixer) de l'optimum absolu.

\section{Matériaux utilisés}

Deux types de matériaux se rencontrent sur les grosses adductions de la S.C.P. : l'acier et le béton armé précontraint.

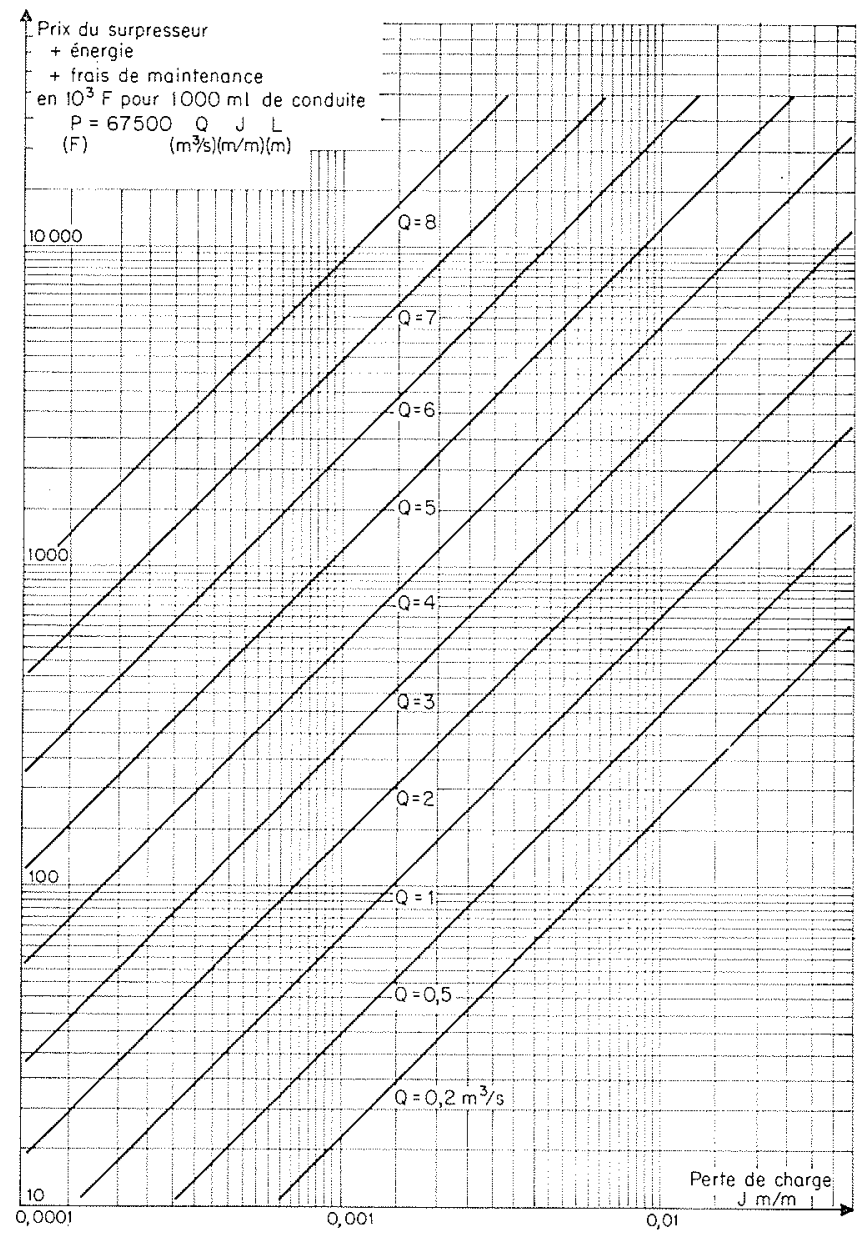

$5 /$ Adduction par canalisations. Installation éventuelle de surpresseurs.

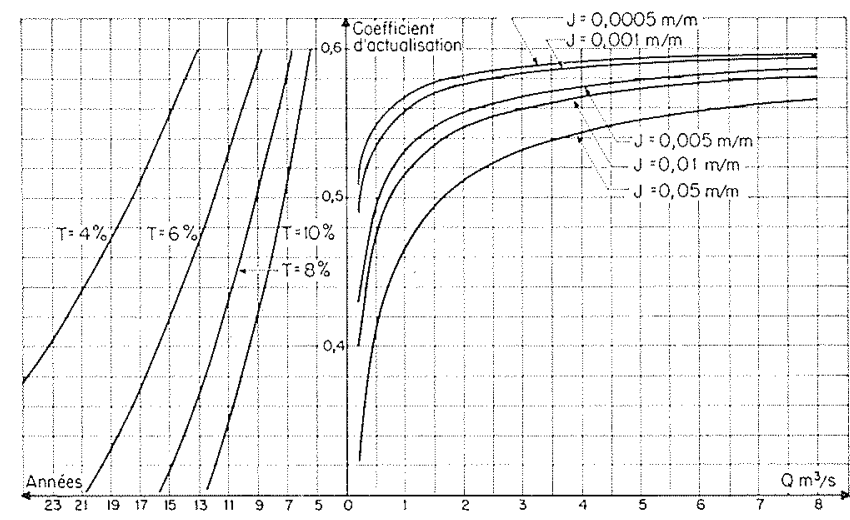

6/ Date économique du doublage en fonction du taux d'actualisation. 
Cas de l'adduction sans

réserve de compensation

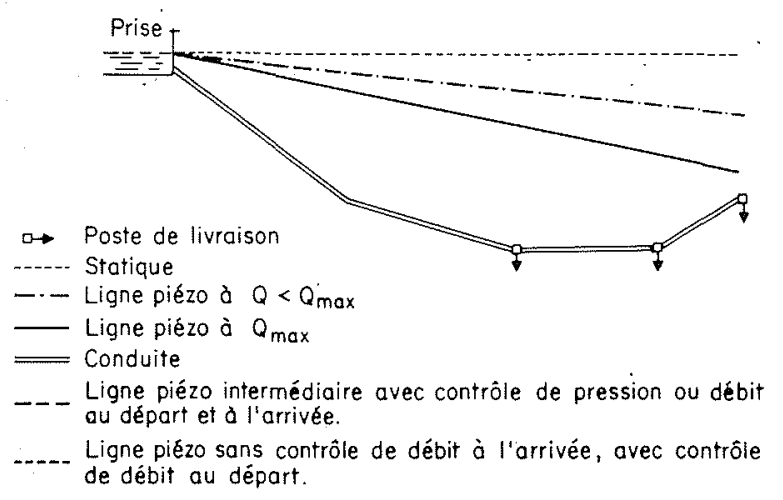

Cas de l'adduction avec

réserve de compensation

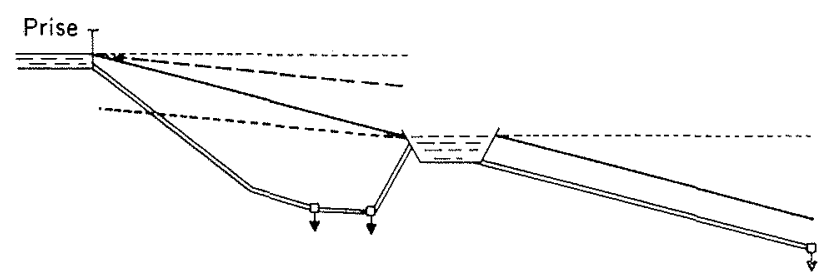

7.8/ Fonctionnement en régime permanent.

Le choix résulte, la plupart du temps, de considérations économiques plutôt que techniques.

Cependant quelques points particuliers méritent d'être soulignés.

\section{Conduite acier}

Les problèmes posés par les conduites acier sont ceux de l'épaisseur des tôles, de la forme des tubes et des revêtements.

- L'épaisseur est calculée de façon à ce que :

- la contrainte dans l'acier sous l'action de la pression statique intérieure ne dépasse pas 0,5 à 0,75 fois $1 \mathrm{a}$ limite élastique de l'acier;

- la déformation du tube vide sous l'action des charges et surcharges extérieures n'excède pas 2 à $5 \%$.

Cette pratique des calculs découle des travaux effectués par divers auteurs ou organismes (American Water Work Association, MM. Prévost et Dreyfus).

La conduite de Berre-Sud $(\varnothing 1300 \mathrm{~mm})$ a été réalisée en acier E 26 de $9 \mathrm{~mm}$ d'épaisseur soit un rapport e/ $D=1 / 145$ et nous avons pu contrôler, par des essais en vraie grandeur avec différents types de remblais, que la déformation du tube n'était pas supérieure aux tolérances.

- La forme des tubes (tolérance sur le périmètre et écart de dimension entre deux diamètres perpendiculaires) doit permettre la soudure bout à bout sans chaudronnage important.
- Les Revêtements doivent protéger le métal contre l'action de l'eau transportée et contre celle du terrain.

L'eau transportée par la S.C.P. n'est pas agressive et, à plusieures reprises, il a été tenté, avec succès, la suppression des revêtements intérieurs.

Par contre, la protection extérieure est indispensable. Elle est réalisée, d'une part en entourant la conduite d'un revêtement à base de brai de houllle et de laine de verre et, d'autre part, en réalisant une protection cathodique.

Après avoir constaté l'insuffisance, sur les conduites de gros diamètre $(\varnothing \geqslant 700 \mathrm{~mm})$ de certains revêtements du commerce qui se fissurent au niveau de la génératrice supérieure sous l'action des mouvements relatifs terre-tuyau, la S.C.P. exige des revêtements plus résistants, armés par une toile (et non un fil), qui ont donné satisfaction jusqu’à ce jour.

\section{Conduite en béton armé précontraint}

Les problèmes posés par les chantiers de conduites en béton armé précontraint sont peu nombreux.

On peut citer, toutefois, le manque de souplesse de ce matériau, dont les pièces spéciales sont fabriquées en usine, et les risques de casse des tulipes lorsque les déplacements angulaires des tuyaux entre eux dépassent les valeurs tolérées par le constructeur. Pour éliminer ces risques, la S.C.P. niaccepte pas que les déplacements angulaires soient réalisés autrement que par des pièces spéciales, laissant ainsi plus de marge en cas de mouvement de terrain.

\section{Fonctionnement hydraulique Régimes transitoires. Protections Sécurités}

\section{Fonctionnement en régime permanent}

Toutes les adductions de la S.C.P. fonctionnent à la demande, en commande par l'aval, et l'utilisateur dispose à tout instant de la pression et du débit qu'il a souscrits.

Chaque client est relié à l'adduction par l'intermédiaire d'un poste de livraison qui limite le débit et la pression aux valeurs de consigne affichées. Un ensemble, vanne papillon, régulateur PDI (proportionnel, différentiel et intégral), capteur de pression et débitmètre, assure la régulation.

En régime permanent, la ligne piézométrique de la conduite est ainsi maintenue au-dessus de la cote piézométrique garantie en chaque point de livraison, qui n'est atteinte que lorsque le débit maximum est appelé simultanément par tous les utilisateurs branchés sur l'adduction (fig. 7).

Le fonctionnement est un peu différent lorsqu'une réserve de compensation est intercalée entre la prise d'eau et les postes de livraison. Le niveau du plan d'eau constitue alors un point obligé de la ligne piézométrique, ce qui entraîne des variations de débit à l'extrémité de la conduito en fonction :

- des fuctuations de prélèvement des utilisateurs;

- et des variations du niveau de la réserve (qui provoquent une ouverture du robinet à flotteur et une perte de charge plus ou moins importante).

\section{(6p)}


Dans certains cas, il est nécessaire d'équiper l'arrivée d'un dispositif plus élaboré qu'un simple robinet à flotteur, de manière à contrôler, comme sur un poste de comptage, le débit et la pression.

Cette solution doit être envisagée lorsque les possibilités de transport de la conduite sont supérieures au débit fourni par la source, ce qui se produit généralement pendant toutes les années précédant le stade exhaustif, quand le maître d'ouvrage limite volontairement ses prélèvements (pour des raisons financières ou hydrologiques). Elle doit également être envisagée lorsque le passage de points hauts, ou le respect de certaines cotes obligées, nécessitent un niveau piézométrique élevé à l'amont de la réserve (fig. 8).

Ces deux conditions ont joué pour l'adduction de BerreSud où :

- d'une part, le prélèvement sur le canal de Marseille (objet d'un accord avec la Société des Eaux de Marseille) est limité par des modules à masque et adapté à l'évolution des besoins;

- d'autre part, la desserte d'un client, situé juste à l'amont de la réserve, nécessite pendant quelques années le maintien (avant l'installation d'un surpresseur) d'une cote élevée en ce point.

\section{Régimes transitoires. Protections. Sécurités.}

Deux principes sont retenus pour définir la protection des conduites contre les coups de bélier :

- Eviter, en fonctionnement normal, les manouvres rapides de sectionnement.

Dans ce but, celles-ci sont toutes équipées de servomoteurs électriques dimensionnés pour que leur temps de fermeture n'engendre pas de surpressions excessives (en général 2 bars).

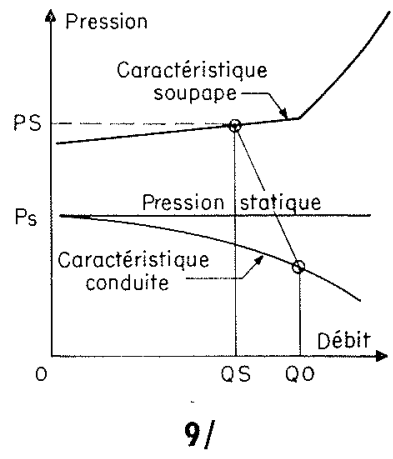

Le calcul du temps est réalisé à l'aide de la formule de Michauds :

$$
\Delta H=2 L V_{0} / g t
$$

où $t$ est le temps de fermeture effective de la vanne, égal au tiers du temps total de fermeture,

et $\Delta H$ la surpression maximum prise égale à 2 bars.

- En dehors des cas de fonctionnement normal, il peut se produire des accidents entraînant une coupure brutale du débit (par exemple avarie de vanne de sectionnement ou fausse manœuvre sur les postes de comptage).

Ces événements, de faible probabilité, sont tout de même à prendre en considération dans le cas d'une utilisation continue n'admettant pas de coupure de longue durée.

On recherche alors à limiter la surpression à la valeur de la pression d'essai en tranchée, qui varie en fonction du matériau utilisé $(1,5$ fois la pression maximum de service pour l'acier, la pression de service +4 bars pour le béton précontraint).

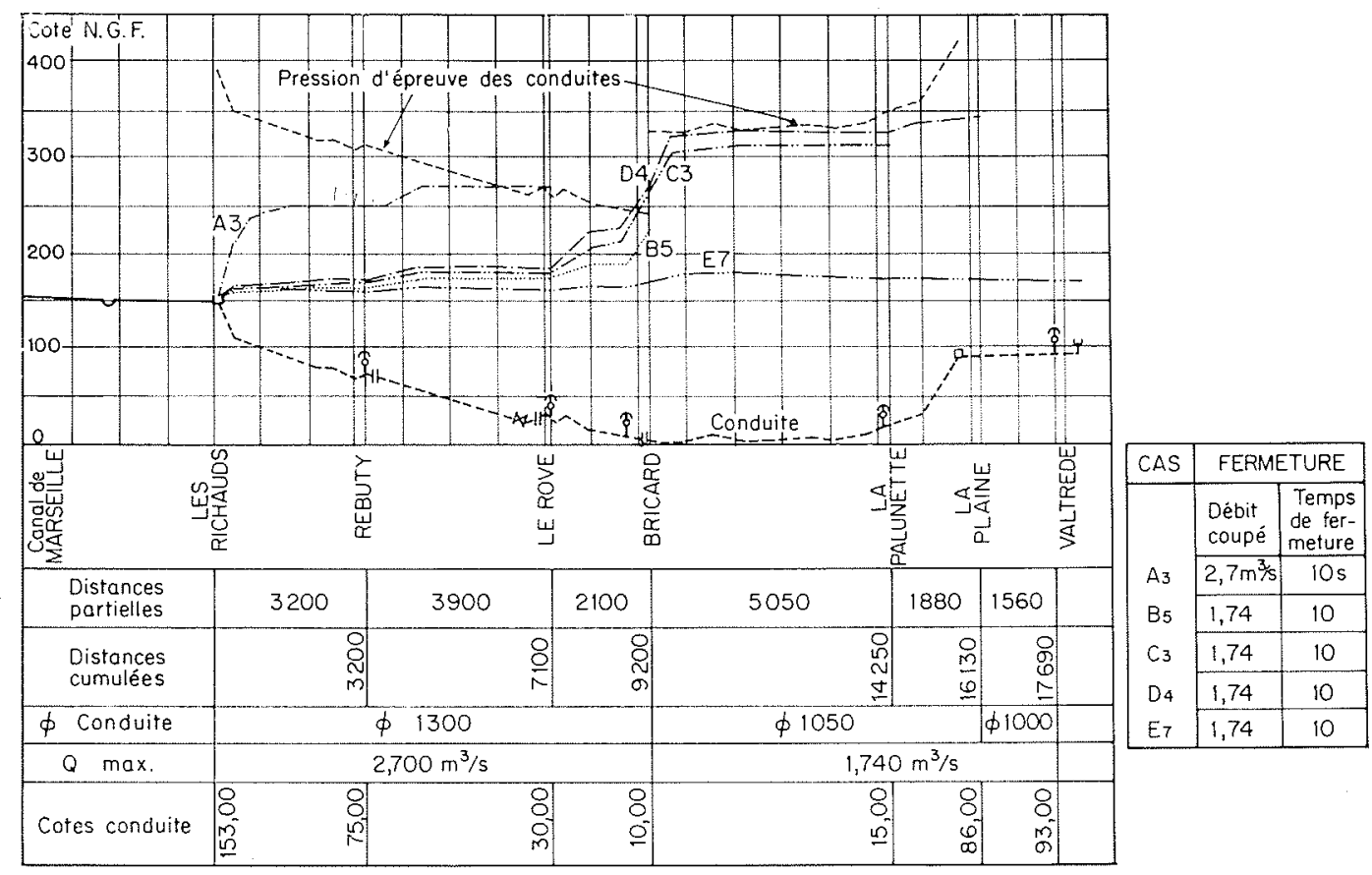

10/ Fermeture brutale des vannes. 
Ce type de protection est assuré par des soupapes de décharge.

D'autre part, la protection contre les dépressions est assurée par des clapets d'entrée d'air placés à l'aval de chaque vanne de sectionnement.

Les soupapes de décharge sont calculées individuellement pour protéger la vanne à l'amont de laquelle elles sont placées. Ces soupapes évacuent ainsi un débit légèrement inférieur à celui de la conduite sous la charge correspondant à la pression d'épreuve (fig. 9).

Ces soupapes sont calibrées pour :

- être étanches sous une pression voisine de la statique $+5 \%$

- évacuer un débit légèrement inférieur à celui de la conduite sous une charge correspondant à la pression d'épreuve en tranchée.
Quand toutes les soupapes sont ainsi dimensionnées, on vérifie, par un calcul sur machine, que la protection envisagée sur l'ensemble de la conduite est correcte, et qưil n'existe pas de point particulier du profil en long où la surpression, engendrée par la fermeture accidentelle et brutale d'une quelconque des vannes, ne dépasse pas la pression d'épreuve en tranchée.

Nous donnons, (fig. 10), les résultats obtenus pour la conduite en acier de Berre-Sud, où la pression d'épreuve en tranchée était de 33 bars pour le tronçon en $1050 \mathrm{~mm}$ et 24 bars pour le tronçon en $1300 \mathrm{~mm}$.

On constate que la surpression est égale à la pression d'essai en tranchée sur une grande partie de la conduite.

Nous estimons que, pour des cas de régime transitoire aussi exceptionnels, il n'est pas souhaitable de multiplier les coefficients de sécurité et, qu'en tout état de cause, l'acier travaille encore dans ces conditions sous un taux compris entre 66 et $80 \%$ de sa limite élastique.

\section{Annexes}

\section{1 - Prix des conduites en fonction du débit et de la perte de charge}

Le prix d'un mètre linéaire de conduite fourni, posé, y compris tous les accessoires (pièces spéciales, robinetterie, génie civil), les honoraires d'études, les taxes et les servitudes foncières, en fonction de son diamètre $D$, s'ajuste généralement à une loi de la forme :

$$
p=A+A^{\prime} D^{\beta} \quad(D, \text { diamètre })
$$

soit pour $L$ mètre :

$$
P=A L+A^{\prime} D^{\beta} L
$$

D'autre part, la perte de charge sur un tronçon s'exprime par :

$$
H=B Q^{2} D^{\alpha} L
$$

avec $: \beta=$ coeff.; $Q=$ débit; $D=$ diamètre; $L=$ longueur.

En éliminant le diamètre dans ces deux formules, on obtient :

$$
P=A L+A^{\prime}(B / H)^{K-1} C^{K}
$$

avec

$$
K=(\alpha-\beta) / \alpha
$$

et

$$
C=Q^{2 \beta /(\beta-\alpha)} L
$$

Dans les conditions économiques de 1973, nous avons :

$$
\begin{aligned}
& P=56+1115 D^{1,73} \\
& (P \text { en } \mathrm{F} ; D \text { en } \mathrm{m})
\end{aligned}
$$

D'autre part, en admettant comme formule de perte de charge celle de Vibert, nous avons :

$$
H / L=1,66 \cdot 10^{-3} Q^{2} D^{-5,25}
$$

avec : $H=$ perte de charge en $\mathrm{m} ; L=$ longueur du tronçon en $\mathrm{m} ; Q=$ débit en $\mathrm{m}^{3} / \mathrm{s} ; D=$ diamètre en $\mathrm{m}$.

Ce qui donne :

$$
\begin{gathered}
C=Q^{\frac{2 B}{\beta-\alpha}} L=Q^{\frac{2 \times 1.73}{1,73+5,25}} L=L Q^{0,51} \\
K=\frac{\alpha-\beta}{\alpha}=\frac{-5,25-1,73}{-5,25}=1,33 \\
b^{\mathrm{K}-1}=\left(1,66 \cdot 10^{-3}\right)^{0,33}=0,102 \\
C^{\mathrm{K}}=\left(L Q^{0,51.1}\right)^{1,33}=L^{1,33} Q^{0,68}
\end{gathered}
$$

et

$$
P=56 L+1115\left(0,102 / H^{0,33}\right) L^{1,33} Q^{0,68}
$$

$$
P=56 L+114 \quad \overline{L^{1,33}} Q^{0,68}
$$

\section{2 - Calcul de la date économique du doublage des conduites}

Dans les conditions économiques actuelles et d'après l'annexe $\mathrm{N}^{\circ} 1$, le prix (en $\mathrm{F}$ ) d'une adduction en fonction de sa longueur $L$ (en $\mathrm{m}$ ), de son débit $Q\left(\right.$ en $\mathrm{m}^{3} / \mathrm{s}$ ) et de la perte de charge disponible $H$ (en $\mathrm{m}$ ), s'exprime par la formule :

$$
P=56 L+114 \quad \frac{L^{1,33}}{H^{0,33}} Q^{0,68}
$$


Etudions dans quelle condition la solution du doublage est plus économique que la solution d'une conduite unique posée à l'année 0 :

Le prix de la conduite exhaustive est :

$$
P=56 L+114 \frac{L^{1,33}}{H^{0,33}} Q^{0,68}
$$

Celui de la conduite doublée posée en $1^{\text {re }}$ tranche :

$$
\begin{aligned}
P & =56 L+114 \frac{L^{1,33}}{H^{0,33}}\left(\frac{Q}{2}\right)^{0,68} \\
& =56 L+71 \frac{L^{1,33}}{H^{0,33}} Q^{0,68}
\end{aligned}
$$

Celui de la conduite dédoublée posée en $2^{\circ}$ tranche :

$$
P=C\left(56 L+71 \frac{L^{1,33}}{H^{0,33}} Q^{0,68}\right)
$$

$C=$ coefficient d'actualisation

Calculons la valeur de $C$ pour que la solution de deux conduites dédoublées soit avantageuse :

$$
\begin{aligned}
(1+C)\left(56 L+71 \frac{L^{1,33}}{H^{0,33}} Q^{0,68}\right) & \\
& <56 L+114 \frac{L^{1,33}}{H^{0,33}} Q^{0,68} \\
\left(56 L+71 \frac{L^{1,33}}{H^{0,33}} Q^{0,68}\right) C & <43 \frac{L^{1,33}}{H^{0,33}} Q^{0,68}
\end{aligned}
$$$$
C<\frac{43\left(L^{1,33} / H^{0,33}\right) Q^{0,68}}{56 L+71\left(L^{1,33} / H^{0,33}\right) Q^{, 068}}
$$

Si l'on appelle $J$ la perte de charge par $\mathrm{m}$ :

$$
J=H / L
$$

l'équation (1) s'écrit :

$$
C<\frac{43\left(L / J^{0,33}\right) Q^{0,68}}{56} \frac{,^{0}+71\left(L / J^{0,33}\right) Q^{0,68}}{(-7)}
$$

$$
C<\frac{43\left(Q^{0,68} \mathrm{~m}^{3} / \mathrm{s} / J^{0,33} \mathrm{~mm}\right)}{56+71\left(Q^{0,68} / J^{0,33}\right)}
$$

\section{3 - Calcul des dépenses d'énergie de pompage}

La dépense d'énergie annuelle s'exprime par :

$$
P=\frac{V \cdot H \cdot p}{102 \cdot \eta \cdot 3600}
$$

avec $V$ volume d'eau élevé en litres; $H$ hauteur de refoulement en mètres; $p$ prix du kWh en francs; $\eta$ rendement de l'installation.

En première approximation et pour dégrossir le problème, on peut admettre que :

$\eta=0,70 ; p=0,08 \mathrm{~F}$ (prix moyen pondéré d'un $\mathrm{kWh}$ souscrit et utilisé toute l'année).

D'autre part, pour simplifier les calculs et ne pas introduire un nouveau paramètre d'étude, $V$ sera calculé en fonction du débit.

Pour une installation fonctionnant en régime continu toute l'année, nous avons :

$$
V=3,15 \cdot 10^{10} Q \quad\left(\text { avec } V \text { en litres; } Q \text { en } \mathrm{m}^{3} / \mathrm{s}\right. \text { ) }
$$

La dépense d'énergie annuelle devient ainsi :

$$
P=\frac{3,15 \cdot 10^{10} \cdot Q H \cdot 0,08}{102 \cdot 0,70 \cdot 3600}=9,80 \cdot 10^{3} Q H
$$

arrondi à $P=10^{4} Q H$

\section{Discussion}

Président : M. H. LORIFERNE

M. le Président souligne, en ces termes, l'intérêt de la communication qui vient d'être présentée :

Je remercie beaucoup $M$. Blanc de son exposé et de l'étude très complète qu'il a faite sur les différents problèmes qui se posent pour le canal de Provence, mais qui sont directement extrapolab'es aux adductions d'eau potable. D'ailleurs, un certain nombre d'idées seraient, non pas à transposer, mais à étudier dans les mêmes conditions pour ce qui concerne les réseaux d'assainissement, en particulier pour les surpressions, la réalisation par tranches de certaines canaljsations; M. Rousset est tout indiqué pour se livrer à ce vaste travail.

La discussion est ouverte.
On pourrait, observe $M$. RousseT, se poser également la question de savoir s'il faut ou non faire des bassins de retenue dans les réseaux d'assainissement; $M$. Blanc a montré dans quel'es conditions ce système était avantageux dans les réseaux d'irrigation.

Ce problème des bassins de retenue, dit M. le Président, est lié à l'utilisation de réseaux dits séparatifs. Il se pose lorsque comme pour la nouvelle ville de Trappes, implantée sur le plateau qui s'étend à l'ouest de Paris - on se trouve très loin de rivières à débit important et que le rejet des eaux pluviales ne peut être envisagé dans les petits ruisseaux du voisinage.

Je ne dis pas que les conclusions auxquelles on aboutit, dit M. BLANC, sont applicables aux réseaux d'assainissement, mais il y a certainement une démarche de l'esprit qui est la même dans les 
deux cas, tout en pouvant conduire à des conclusions différentes. II est certain que, dans une ville ou une cité très organisée - à Paris ou dans sa ban'ieue, par exemple - il faut poser tout de suite la canalisation définitive; il n'est pas question d'envisager son doublement à moyen terme cat, entre temps, d'autres auront pris la place.

Dans le cas d'un réseau d'assainissement pluvial, il n'y a guère d'incertitude sur la demande, remarque $\mathrm{M}$. BLANC.

Dans ce cas également, répond $M$. le Président, il y a une évolution de la demande dans le temps qui est difficile à prévoir.

Pour les eaux pluviales, l'influence de l'urbanisation sur les flots d'orage pose un problème; pour les eaux usées, le développement de la consommation d'eau à laquelle M. DARGENT a fait allusion tout à l'heure, fait qu'on ne sait pas très bien la quantité d'eau qui sera consommée - donc rejetée à l'égont — dans dix, quinze ou vingt ans.

Les calculs d'optimisation proposés par M. BuaNC, observe M. RÉMÉNIÉRAS, s'entendent à «francs constants», ils devraient faire l'objet de quelques corrections - différentes suivant le mode de financement adopté - pour tenir compte de l'érosion monétaire..

M. le Président clôt la discussion et lève la séance à $18 \mathrm{~h} 15$, en remerciant tous les auteurs de communications, ainsi que les personnes qui sont intervenues dans la discussion.

\section{Abstract}

\section{Design and operation of high-discharge water supply duct systems under pressure}

\section{Introduction}

Water supply systems above a certain size contain the following sub-systems, from the river intake to the final user :-

1. Upstream conveying system.

2. The supply system proper.

3. The distribution system.

The upstream conveying system consists of high-discharge canals or tunnels (depending on topography).

The distribution system is a ramified pressure pipe network anding at the users' oftakes.

Between these two sub-systems, there is almost invariably a third, which can be considered to constitute the supply system proper. This comprises appreciable lenghts of large-diameter pipe frequently operating under high pressure and conveying flows of a few cubic metres per second. System features depend on local topography; if the upstream conveying system is at a sufficient height, the pipe flow is under gravity, and if not, pumping is resorted to.

The S.C.P. Owns a number of sub-sistems of this type, with a total pipe length of approximately $65,000 \mathrm{~m}$. Others p'anned include "Berre-Ouest", "Berre-Nord", and extensions to the

"Aix-Nord", "Toulon-Est" and other existing systems (see Fig. 1).

Specific design problems for all these projects are discussed in this report.

\section{Pipe runs}

Pipe runs must be as straight as possible, because of the high cost per lineal metre pipe length.

\section{Design discharge}

When demand varies considerably during a peak day it often costs less to install a compensation reservoir and operate the water supply system continously. Per kilometre pipe length and compensating storage costs can be calculated rapidly from Figs. 3 and 4

\section{Available head. Requirement for booster station}

Since pipe cost deoreases with available head loss (Fig. 3), addition of a booster station to increase this head may result in a more economical system. This is unlikely for a system in continuous operation, but where a booster station is required to get the water over a high point, the economic optimum generally consists in increasing the strict minimum geometric head required. The cost of a booster station and corresponding power expenditure can be evaluated from Fig. 5.

\section{Stagewise impementation. Duplication of pipes}

A'though implementation of a water supply system in two stages (duplication of pipes) is very difficult in practice, it undoubtedly is an attractive economic proposition for systems expected to supply a steadily increasing demand. The time at which duplication is most economical can be calculated from Fig. 6.

\section{Materials used}

Steel and prestressed concrete.

\section{Operation}

The water supply systems described operate "on demand", under downstream contro!. The delivery stations limit the rate of flow called for to the proportions subscribed for by the users; some control arrangements maintain the piezometric gradient below the guaranteed contract value (see Figs. 7 and 8 ).

Al1 installations are protected against transient effects (e.g. hammerblow).

Fig. 10 shows results obtained with a large S.C.P.-owned system. 